\title{
Piedra artificial porosa a partir de residuos de rocas ornamentales adaptable a obras de construcción y restauración patrimonial
}

\author{
J.A. Durán Suárez ${ }^{(1)}$ y A. García Casco ${ }^{(2)}$ \\ (1) Departamento de Escultura-Restauración. Universidad de Granada. giorgio@ugr. es \\ (2) Universidad de Granada e Instituto Andaluz de Ciencias de laTierra, CSIC-UGR. agcasco@ugr. es
}

\section{RESUMEN}

La restauración arquitectónica patrimonial emplea la técnica de reintegración volumétrica en los soportes pétreos gravemente deteriorados. En la utilización de morteros de restauración y recrecido se requiere controlar aspectos importantes como: similitud cromática y textural respecto del entorno pétreo adyacente, menor o igual resistencia mecánica e igual o mayor porosidad/permeabilidad, respecto a la piedra original. Este último aspecto es particularmente complicado pues también se requiere que la distribución porcentual de rangos de poro no sea discordante con la que presenta el material pétreo original.

A través de la patente de invención (con números de publicación ES2187245 A1 y B1 16. 06. 2004), con titularidad de la Universidad de Granada, se ha logrado una excelente piedra artificial porosa aplicable a trabajos decorativos en construcción y un mortero de restauración de materiales pétreos con porosidad controlada. Ambos están constituidos, en gran parte, por residuos de rocas ornamentales por lo que se da un uso concreto a este tipo de subproductos difícilmente reutilizables. En este trabajo presentamos el procedimiento para obtener estas piedras artificiales y morteros porosos con fines constructivos, decorativos y restauradores, a partir de la mezcla controlada de áridos de rocas ornamentales, aglomerantes inorgánicos, orgánicos, o sus mezclas y generador de porosidad. Tras el fraguado y endurecimiento se pone de manifiesto uno de los aspectos más relevantes del producto, que se basa en la generación de un complejo sistema poroso conectado gracias a la incorporación de poliestireno expandido molido, el cual se activa tras la puesta en obra mediante la aplicación de disolventes orgánicos.

Palabras clave: material construcción, permeabilidad, porosidad conectada, piedra artificial, restauración monumento), residuos, reciclado.

\section{Artificial porous stone from of ornamental rock waste adaptable for civil con- struction and heritage restoration}

\begin{abstract}
The technique of volumetric reintegration in severely deteriorated stone materials is of major importance for the restoration of architectural heritage. In using mortars for restoration it is important to control aspects such as the colour and textural similarity in relation to the adjacent stone, minor or equal strength and equal or greater porosity/permeability with respect to the original stone. This latter aspect is of particular complexity because the percentage distribution of pore-size ranges should not differ from that of the original stone material.

The invention patent (with numbers of publication ES2187245 A1 and B1 16. 06. 2004), owned by the University of Granada, allows the production of excellent porous stones for decorative work in construction and mortar for restoration of stone materials with controlled porosity. Both materials consist of ornamental rock waste, so that a particular recycling purpose is given to this type of material which is difficult to reuse. In this paper we present the manufacturing process of these artificial stones and porous mortars with decorative and restorative purposes, from the controlled mixture of aggregates of ornamental rocks, organic and inorganic binders and generators of porosity. Once the curing and hardening has been done it is worth noting that one of the most important aspects of the product is the generation of a complex connected porous system as a result of the incorporation of crushed expanded polystyrene, which is activated after hardening through the application of organic solvents.
\end{abstract}


Durán Suárez, J.A. y García Casco, A., 2017. Piedra artificial porosa a partir de residuos... Boletín Geológico y Minero, 128 (2): $437-450$

Keywords: construction materials, permeability, connected porosity, artificial stone, monument restoration, waste, recycling.

\section{ABRIDGED ENGLISH VERSION}

\section{Introduction and methods}

Developments in the manufacture of artificial stone and other building elements are decisive for activities such as architecture, industrial design and construction. Ready-to-use products and mixtures designed for subsequent applications in construction are available on the market. All these products have good quality standards and optimum finish, although they may also have some disadvantages, such as a lack of connected porosity (Casabó, 1958; García \& Gómez, 1995).

The artificial stone industry offers low-cost building materials (flooring, prefabricated regular and serial parts, wall coverings, etc.) with appropriate mechanical and aesthetic properties. In addition, the availability of special high-porosity mortars with the main purpose of reducing weight whilst acting as thermal and acoustic insulators (mortar or cellular concrete, low density, refractory, resistant to radiation, self-levelling, etc.) is remarkable. However, their greatest disadvantage lies in the fact that they have an un-connected porous system.

Therefore, it is important to seek the production of mixtures or ready-to-use products whose physical properties include elevated connected porosity in addition to appropriate mechanical properties. It is also important that these products use waste materials resulting from cutting ornamental stones in order to devise procedures with additional significant economic and environmental advantages.

This paper presents a manufactured solid porous product made mainly from recycled waste rock materials which simulates different types of ornamental stones and can be adapted to construction and heritage restoration (patent publication ES2187245 A1 and B1 numbers 16/05/2003), owned by the University of Granada), and a mortar (kit) that can also be used for both proposals. A special application is the restoration of porous stones used in artistic historical buildings, when they suffer from capillary infiltration problems. The basic intention of the product design is that the added material does not interfere negatively with the original work, guaranteeing the success of the restoration (Santiago, V. 1995. Durán \& García-Casco, 2003).

\section{Results and discussion}

The mixture is made up of four components in variable proportions: stone aggregates, preferably produced after crushing building stones, cement of different types including hydraulic and non-hydraulic varieties, and additives such as chromophores and a porosity generator made of crushed expanded polystyrene (EPS) forming grains of variable size.

The artificial stone can be prepared as a pre-manufactured material after the use of casts. It can also be prepared as a ready-to-use piece or as a mortar (adjustable kit) to be directly applied as a raw material with the use of a mould or not. These mixtures can be designed to order, depending on the purpose (fabric or decorative elements, restoration...), allowing the production of building elements with the desired physical and aesthetic properties. Any other additive can be combined in the mixture for achieving particular purposes except, obviously, those that may damage the mixture and those that may hamper the production of porosity. Porosity is generated after the application of organic solvents (toluene, xylene, acetone...) once the product is forged, hardened and completely dry.

The amount of solvent to be used is lowest in the case of pre-manufactured pieces and decorative mortars, but it increases significantly in the case of restoration purposes because the connected porosity must be attained throughout the piece/added mortar. These products allow the application of humidity protectors, hardeners/strengtheners, and varnishes that enhance the final product, in addition to allowing finish, surface treatment and polish.

The proportions of the components can be varied at will as a function of the use and characteristics of the building or restoration stone to be simulated. Thus, the volume proportion 1:2:3 of cement:aggregates:polystyrene, with carbonate rock (e. g., marble, micritic limestone) grains of building material ( $<1 \mathrm{~mm}$ in size) and large grains of polystyrene $(1 \mathrm{~mm}$, and up to $1 \mathrm{~cm}$ or larger), allows the reproduction of strongly porous and cavernous rocks (50 \% volume) such as travertine (figure 1). Lower proportions of cement, e. g., 1:3:1 cement:aggregates:polystyrene, with a finer grain size of the pore-generator polystyrene and sieved carbonate or siliceous (or a mixture of both) building stone sand $(1-7 \mathrm{~mm}$ ) allows the reproduction of calcareous and siliceous sandstone satisfactorily (Fig. 2). The nature of the cement can be selected from hydraulic lime or organic resins including epoxy or polystyrenes. The cement resulting from mixing salt-lacking non-hydraulic and hydraulic lime is appropriate for mortars for restoration. 
Durán Suárez, J.A. y García Casco, A., 2017. Piedra artificial porosa a partir de residuos... Boletín Geológico y Minero, 128 (2): $437-450$

The process of obtaining connected porosity consists of adding an organic solvent to the mixture in order to dissolve the EPS. This reaction is fast, as long as the material is completely dry, and the progress is rapid from the surface of the product to its interior (Fig. 3). The variations in pore volume and pore-size distribution achieved (Fig. 4) in the process of applying the artificial stone for the reconstruction of stony material of the architectural heritage are slight and conform to international standards (Rossi-Doria, 1986). The total porosity and pore-size distribution (obtained with a Micromeritics Autopore IV porometer, operated at 33000 psi) of travertine and artificial rock samples simulating travertine show that similar to higher porosity can be achieved in the latter (30\% vs 35-49\%), whilst the pore-size distribution is similar in both types of material.

The experimental decomposition of EPS (Table 1) shows that toluene has a greater effect in removing EPS than acetone (31 and $51 \mathrm{~g}$ of EPS removed by $100 \mathrm{ml}$ of acetone and toluene, respectively), the reaction being faster in the first stages (Fig. 5).

The benefits of the effective control on the porosity and pore-size of the artificial stone for the alteration of the added material once it is set in the building and suffers atmospheric stress should be noted These benefits are achieved after producing a given grain-size distribution of the polystyrene aggregates that allows the reduction of the capillary infiltration of solutions from the basement. In fact, the transfer of water solutions through open macropores, in contact with the external atmospheric environment, allows the rapid evacuation of the solutions by means of transpiration.

Two basic methods of application of the invention are described below: a) as ready-to-use objects and b) as mortars to be applied in situ (adjustable kit). In the first case, the proportions of cement, aggregates, polystyrene and pigments must be evaluated after appropriate test controls of the properties of the stone to be simulated, including mechanical strength, porosity and chromatic properties (Durán et al., 2000). All the components are mixed in a concrete mixer adding up to $50 \%$ water, and the mixture is poured into the cast once it reaches a plastic state. To simulate the layering of some stones, the mixture should be poured sequentially. After first hardening, the added material should be hydrated to ensure an optimum hydraulic hardening and the removal from its mould. Finally, after the product is dry, it is plunged into a bath containing the organic solvent for a few seconds and then dried. The adjustable mortars are made in the same manner, except that the mixtures are prepared dry and kneaded in situ at the construction site.

The plaster should not be shaken, as the pore-generator agent (EPS) flows up in the mixture due to density contrast (Payá, 1985). The time for curing the hydraulic mixtures is ca 1 month. Once dry, the reactive solvent (toluene, acetone) is applied to the product in order to decompose the grains of polystyrene (EPS) of the mixture, forming the characteristic and novel pore system of the porous artificial stone (NORMAL 4/0, 1980).

\section{Conclusions}

The porous stone presented in this paper has shown an excellent performance in construction and architectural heritage restoration work since its patent registration. Whether as a prefabricated element or as adaptable work kit, the cost of such products is much less than that of natural stone. The procedure presented also allows the recycling of waste produced by the ornamental rock industry, thus adding an application for recycling these products.

The great innovation of this product lies in its use in restoration work on architectural and monumental heritage, mainly because of its connected porosity system obtained after the inclusion in the mixture of a pore generating agent and subsequent removal by means of adding solvents. This open porosity is critical for the success of the restoration work of stone exposed to atmospheric stress as it allows infiltrating water solutions to flow out of the stony material in the form of water vapour without causing alterations.

The use of this artificial porous stone has been extensive for the last twenty years of heritage restoration in the city of Granada and its surroundings, including the Nazari period (s. XV) travertine pillars of the courtyard of the Collegiate Church of El Salvador in Granada, the masonry of bioclastic calcarenite of Granada Cathedral (s. XVI), the moulded elements of bioclastic calcarenite in the facade of the San Pablo College in Granada (s. XVII) and the restoration of the colonnade of bioclastic calcarenite in the courtyard of the Chapel of the Royal Hospital in Granada (s. XVI).

\section{Introducción y antecedentes}

La fabricación de piedra o elementos constructivos de manera artificial ha supuesto un avance importantísimo en actividades como la arquitectura, ingeniería, diseño industrial y la construcción, donde son destacables los productos obtenidos totalmente manufacturados o preparados para su posterior puesta en obra con una calidad y acabados óptimos (Casabó, 1958; García y Gómez, 1995). 
Desde hace tiempo el sector de piedra artificial ha puesto en el mercado productos que consiguen materiales de construcción (solerías, piezas regulares y seriadas prefabricadas, placas de revestimiento, etc.) a bajo coste, aunque con adecuadas propiedades mecánicas y estéticas. Sin embargo, la producción de revestimientos y materiales ornamentales está más dirigida a la consecución de manufacturados con óptima relación calidad-precio que a la consecución de productos de especiales propiedades técnicas y estéticas (Fernández, 2013). Hay una amplia gama de materiales que reproducen los veteados y texturados de determinadas rocas de carácter ornamental, aunque no se imiten otros aspectos específicos de estos tipos de rocas como, por ejemplo la porosidad visible, la micro porosidad y su grado de interconexión, y la heterogeneidad del tamaño de poro (Russel, 1927; Ashurst and Dimes, 1977; Zehnder, 1982; Veniale and Zezza, 1987; Esbert y Ordaz, 1985).

De forma paralela, es destacable en el mercado la existencia de morteros especiales con elevada porosidad cuya finalidad es aligerar la carga, a la par que actúan como aislantes térmicos y acústicos, entre los que cabe mencionar los morteros u hormigones celulares, espumosos, de baja densidad, refractarios, resistentes a la radiación, autonivelantes, etc. Este tipo de hormigones y piezas prefabricadas incluyen aditivos diversos, todos ellos con carácter no activo que son determinantes en el producto final manufacturado. Así mismo, una vez fraguada y endurecida la mezcla funcionan como material inerte, no interviniendo en el resultado final de las piezas obtenidas. Estos morteros u hormigones especiales, o los prefabricados obtenidos a partir de estos últimos, presentan la desventaja adicional de no tener la porosidad conectada entre sí, pues no facilitan la evacuación de agua o humedad del subsuelo en forma de vapor de agua, taponándola y generando alteraciones tanto en el propio material, como en el soporte original (e. g. precipitación de sales y eflorescencias, disoluciones, etc.).

Por todo ello, resulta interesante fabricar mezclas o productos acabados cuyas propiedades físicas incluyan una elevada porosidad conectada y al mismo tiempo buenas propiedades mecánicas, proporcionando durabilidad, tenacidad y elasticidad, así como fácil trabajabilidad y puesta en obra, posibilidad de mímesis con diferentes tipos de piedra, acabado visual y estético similar al de las rocas naturales de tipo ornamental, posibilidad de texturado y bajo coste de producción. La piedra artificial porosa que se propone en este trabajo permite reutilizar material de desecho procedente del corte de rocas ornamentales o de minería extractiva, el cual no es fácilmente reci- clable, a la par que supone grandes costos económicos y medioambientales.

Según Eurostat, la industria es el principal sector económico generador de residuos en la mayoría de países europeos, seguido de la construcción, servicios y agricultura (Eurostat, 2009). La industria y la construcción generan el mayor volumen de residuos (83 \%) en la Unión Europea, y el sector industrial produjo casi un $55 \%$ de residuos procedentes de canteras y minería en 2006, cuya acumulación en depósitos comportó graves impactos paisajísticos y desastres ambientales.

Numerosas referencias enfatizan la importancia de la actividad de reciclado de residuos, así como su relativa falta de regulación, con importantes ejemplos de reutilización en movimientos de tierras y en la construcción, en procesos de asfaltado (Akbulut and Gürer, 2007; Castro-Gomes et al. 2006), como áridos en la fabricación de hormigones (Hebhoud et al. 2011; Yellishetty et al. 2008), en la producción de cementos tipo Portland, o de morteros poliestéricos (Mun et al. 2007; Peralbo Cano, 2007; Castro-Gomes et al. 2011) para construcción, diseño o tematización, y puesta a punto de aglomerantes de activación alcalina (geopolímeros) a partir de lodos, basados en sílice-alúmina, procedente de minas y canteras (Torgal et al. 2008; Torgal et al. 2009; Zhang et al. 2011).

El principal objetivo de este trabajo consiste en la consecución de un producto manufacturado sólido poroso a partir del reciclado de los residuos de rocas ornamentales, principalmente, que simula diversos tipos de rocas ornamentales, pudiendo ser adaptado a obras de construcción y restauración patrimonial. Este producto está materializado desde hace veinte años en forma de invención, por los autores de este trabajo de investigación. La patente registrada en la Oficina Española de Patentes y Marcas (con números de publicación ES2187245 A1 y B1 16. 06. 2004), propiedad de la Universidad de Granada, se ha aplicado en numerosas intervenciones patrimoniales desde el año 1994 en una doble vertiente. A nivel constructivo y ornamental, la utilización de elementos de piedra artificial en trabajos de construcción permite conferir a las obras una cierta nobleza, al simular piedras naturales, con un gasto moderado. No obstante, algunas piedras naturales de construcción, como el travertino, las areniscas y las tobas volcánicas, presentan tal grado de porosidad que su simulación artificial no es una tarea sencilla. Un primer objetivo de la presente invención es, precisamente, la obtención de productos de construcción (prefabricados y morteros) económicamente rentables que simulen piedras porosas.

Un segundo objetivo de esta invención lo consti- 
tuye la direccionalidad de este tipo de productos para la restauración de piedras porosas utilizadas en construcciones de carácter histórico-artístico, pues los trabajos de restauración de monumentos implican, en la mayoría de los casos, la aplicación de morteros que remplacen pérdidas de sus elementos pétreos constructivos dañados (e. g. sillares de paramentos, tambores de columnas, frisos, cornisas, revestimientos, etc.). Estos trabajos son necesarios, entre otros, cuando existen problemas de infiltración capilar de soluciones acuosas que ascienden desde el subsuelo y circulan por las partes más bajas de los edificios. El fin de estas intervenciones es múltiple: por un lado, debe sanearse la zona intervenida, retirando el material original deteriorado para evitar un rápido deterioro de las zonas adyacentes $y$, por otro lado, se persigue un fin estético, reemplazando las faltas con un material que reproduzca las características texturales, estéticas y cromáticas del material alterado. Así mismo, se pretende que el material añadido no interfiera negativamente con la obra original, de crucial importancia para garantizar el éxito de la operación restauradora (Santiago, 1995; Durán y García-Casco, 2003; Prado et al, 2009). Por ello, es comúnmente necesario utilizar morteros porosos que permitan la evacuación del agua localizada en el sustrato pétreo que recibe el mortero de restitución, más aún cuando la piedra a restaurar es de por sí porosa (Jiménez, et al. 2009). Sin embargo, elaborar morteros de restauración porosos y permeables, y que simulen estéticamente piedras naturales con huecos interconectados no es una tarea sencilla.

\section{Metodología experimental}

Los materiales empleados parten de la mezcla en cantidades variables de cuatro tipos de componentes: 1) áridos, en general de naturaleza pétrea y preferentemente de procedencia de rocas ornamentales, grava de cantera, grava de río, grava de playa lavada, suelos arenosos y productos de desecho de otras actividades industriales de la roca ornamental, cerámica o siderúrgica. 2) Aglomerantes de tipo aéreos o hidráulicos, como por ejemplo: cales aéreas, yesos, cales hidráulicas, cementos hidráulicos o resinas orgánicas. 3) Aditivos cromóforos, como pigmentos inorgánicos y orgánicos y 4) aditivo generador de huecos o poros, fundamentalmente poliestireno expandido de diferentes densidades (EPS), molido, con una granulometría controlada. Los áridos en los casos presentados en este estudio proceden de residuos clasificados de mármol de Macael derivados de procesos de manufactura de la piedra ornamental, y de moltura- ción de roca calcarenita bioclástica, procedentes de las canteras de Escúzar en Granada. El árido procedente de mármol de Macael tiene un tamaño de grano comprendido entre 2 y $0.05 \mathrm{~mm}$, y fue empleado en la fabricación de piedra artificial que imita a travertino y calcarenita bioclástica. El árido molturado procedente de piedra ornamental calcarenita de Escúzar-Granada tiene un tamaño de grano, clasificado mediante cedazos normalizados, comprendido entre 3 y $0.1 \mathrm{~mm}$, se ha utilizado en la fabricación de piedra artificial que imita calcarenita bioclástica, junto con cierta adición de árido de mármol de Macael. La composición del árido de mármol cuya composición mineralógica es de casi un 99 \% de calcita, aunque puede aparecer moscovita procedente de un ligero veteado de laminillas que aparecen en algunos litotipos (la composición química mayoritaria es calcio, carbono con impurezas de silicio, hierro, magnesio y sodio que nunca supera el $0.2 \%$ (Luque, 2011). La composición del árido procedente de calcarenita bioclástica corresponde con la de la roca triturada: fragmentos de organismos fosilizados, procedentes de algas rojas, equinodermos, briozoos, etc, junto a otros minerales del tipo cuarzo, micas y feldespatos, procedentes de los relieves próximos a la depresión de Granada. Todos estos materiales están sustentados por una matriz calcítica (Rodríguez, 1994).

La piedra artificial puede elaborarse como prefabricado de todo tipo: bloques, losas, enchapados, tableros, dovelas, columnas, balaustres, piezas para solería, etc, mediante la utilización previa de moldes o encofrados. También puede estar lista para su puesta en obra como elemento discreto, o como mortero (kit adaptable) aplicable en masa y moldeable in situ con o sin encofrados o moldes, para la generación de enlucidos, repellos, suelos, cornisas, etc.

Las mezclas se diseñan según convenga para los fines perseguidos (elementos resistentes, decorativos o restauradores), lo que permite confeccionar productos con una amplia gama de propiedades físico-mecánicas y estéticas. Por ello, cabe adicionar cualquier otro aditivo oportuno para fines determinados, siempre y cuando no interfiera con el proceso de obtención de la porosidad. Este último proceso, determinante para la obtención de la porosidad conectada, consiste en adicionar disolventes orgánicos (tolueno, xileno, acetona, disolvente universal, disolvente nitrocelulósico, etc.) al producto elaborado una vez fraguado, endurecido y seco, o mediante la aplicación de calor en la superficie, durante el tiempo necesario para retraer el aireante, con pistola de aire caliente a $175^{\circ} \mathrm{C}$. Los reactivos mencionados son inocuos para el material aglomerante y el árido, si bien disuelven los granos de poliestireno expandido, por 
lo que su espacio queda ocupado así por aire, confiriendo al producto la porosidad deseada (tanto en volumen porcentual como en distribución de tamaño de poro). La cantidad de disolvente es mínima para las piezas prefabricadas y los morteros aplicados con fines exclusivamente constructivos, ya que se pretende conferir porosidad, con finalidad estética o visual, a la superficie expuesta del producto. Sin embargo, la cantidad de disolvente es bastante mayor cuando el producto se utiliza para fines restauradores, ya que se pretende conferir porosidad conectada a todo el producto añadido a la obra. La aplicación de calor está indicada para piezas prefabricadas y morteros con fines constructivos sin que se produzcan fisuras 0 grietas, debidas a choque térmico, en la matriz de la piedra artificial.

La mezcla y el material fraguado, tanto para fines constructivos como restauradores, admite la aplicación de protectores de la humedad, consolidantes y esmaltes que mejoren el producto final, así como pulido (Vénuat, 1972; Arredondo, 1977). Para reforzar las propiedades de elasticidad de los productos, particularmente la resistencia a la tensión, puede ser necesaria la utilización de mallas o varillas metálicas (pretensados o no) localizadas en el interior del producto, si bien de forma que permitan el corte (caso de obtención de grandes bloques de material fraguado) de las piezas prefabricadas (Weigler and Sieghart 1974; Páez, 1986; Marí Bernat, 1999; Murcia, et al. 1993).

El análisis de muestras y su valoración está referido a intervenciones de restauración (morteros específicos de restauración) y probetas preparadas en laboratorio (muestras de piedra artificial y muestras de EPS). El número de probetas y mediciones en laboratorio ha sido adecuado en todos los casos. En el estudio realizado para la solicitud de patente de invención se utilizaron un total de 250 muestras entre probetas de laboratorio y aplicaciones de restauración expuestas a la intemperie. Se han empleado diversas técnicas de cuantificación de propiedades físicas de los materiales, como por ejemplo porosimetría de inyección de mercurio, con porosímetro marca Micromeritics Autopore IV, a baja y alta presión, hasta 33000 psi, incrementos de 0.53 psia y ángulo de contacto de $130^{\circ}$. La toma de imágenes macro se ha realizado con una cámara digital Nikon DS46, provista de lente macro AF-S Macro Nikkor de $20 \mathrm{~mm}$. La visualización de huecos y su clasificación, tras la eliminación del generador de poros EPS, se ha realizado con un microscopio estereoscópico marca Leica EZ4 HD provisto de cámara y software Leica LAS EZ, para la medición de los mismos.

\section{Resultados y discusión}

Las proporciones de aglomerantes, áridos, poliestireno y pigmentos pueden ser variadas a voluntad en la mezcla con el fin de adecuar el resultado final a las características mecánicas, porométricas, texturales y cromáticas de la roca ornamental a simular, en construcción, o para ser intervenida durante actividades de conservación-restauración. Con el mismo fin puede variarse la naturaleza del aglomerante, la naturaleza y granulometría del árido, así como la cantidad y granulometría del poliestireno. En todo caso se pueden atender algunas directrices de fabricación de este tipo de productos y de sus aglomerantes (Normas UNE 127748-2:2006; UNE 127748-1:2006; UNE-EN 196-1:2005).

Por ejemplo, la proporción en volumen 1:2:3 de aglomerante:árido:poliestireno, usando árido fino de residuos de roca ornamental $(<1 \mathrm{~mm})$ de naturaleza carbonatada (por ejemplo, de mármol crema, calizas micríticas) y granos de poliestireno gruesos $(1 \mathrm{~mm}$, hasta $1 \mathrm{~cm}$, o mayor), permite reproducir fácilmente rocas muy porosas ( $50 \%$ en volumen) y cavernosas como los travertinos (figura 1).

Menores proporciones, con 1:3:1 de aglomerante:árido:poliestireno, con granulometría más fina del agente generador de poro y arena calibrada procedente de roca ornamental, con tamaño grueso (1-7 $\mathrm{mm}$ ) de naturaleza carbonatada, silícea o mezcla de ambas, permite reproducir areniscas calcáreas o silíceas. En el caso particular de que la arenisca conste esencialmente de fragmentos carbonatados de fósiles, puede obtenerse el árido por molturación controlada de rocas biocalcareníticas (figura 2). Por otra parte, la utilización de árido procedente de rocas volcánicas y granos de poliestireno de tamaño heterogéneo permite simular rocas volcánicas (AENOR, 1996).

Para la utilización de estas mezclas con fines constructivos y decorativos deben adicionarse aglomerantes de alta resistencia, como por ejemplo cementos hidráulicos y/o resinas orgánicas de tipo epoxídico o poliestérico, que puedan mejorar las propiedades mecánicas del producto. Por el contrario, los aglomerantes resultantes de mezclar cal aérea y cal hidráulica son los apropiados para la utilización del producto como mortero de restauración o piedra artificial porosa para reintegración volumétrica en restauración, ya que estos productos no contienen sales solubles (el cemento Portland contiene en torno a un $2 \%$ de yeso). El producto así obtenido presenta una menor resistencia mecánica debido a la utilización de cales, lo cual está indicado para cualquier mortero de restauración (Rossi-Doria 1986). Sin embargo, el curado 

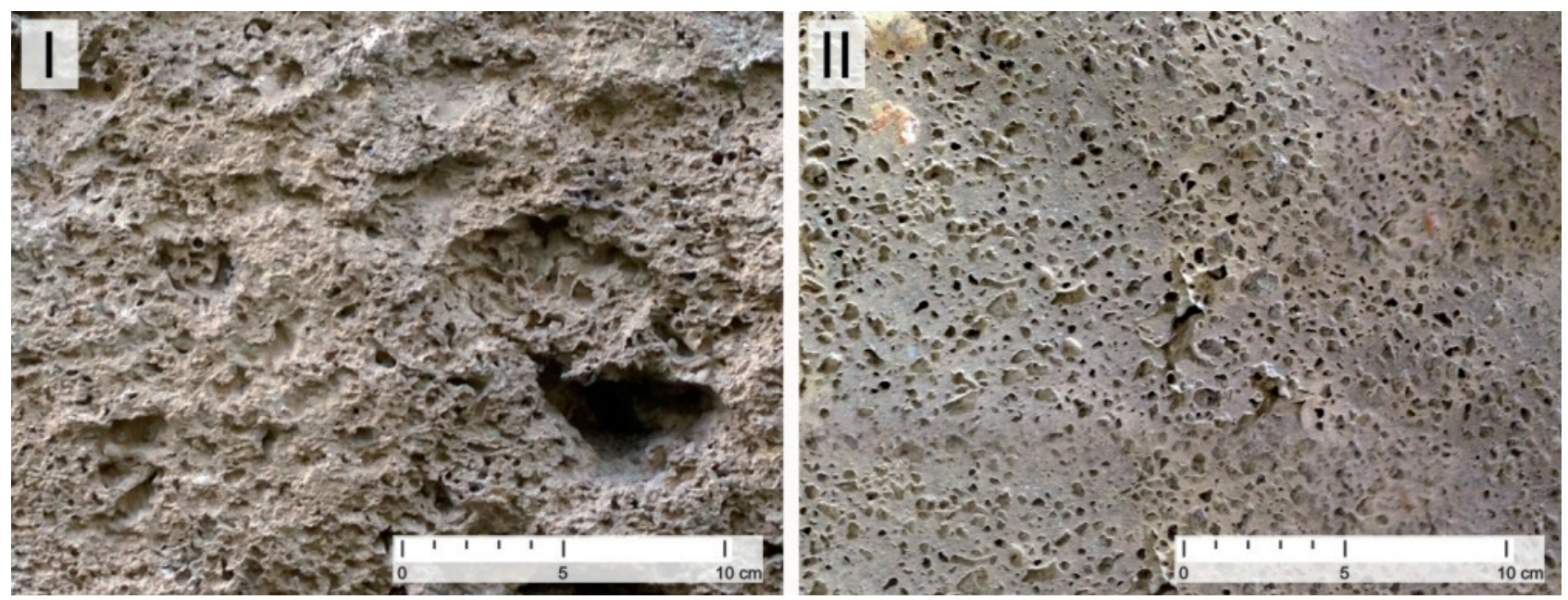

Figura 1. Detalle de piedra travertino, procedente de las canteras de Alfacar-Granada (I) y detalle de piedra artificial porosa que simula el travertino natural (II).

Figure 1. Details of travertine, from the quarries of Alfacar-Granada (I), and of porous artificial stone simulating natural travertine (II).

y endurecido de la cal hidráulica garantiza, por contraposición a los aglomerantes a base de cal aérea sencillos, aceptables propiedades mecánicas a los
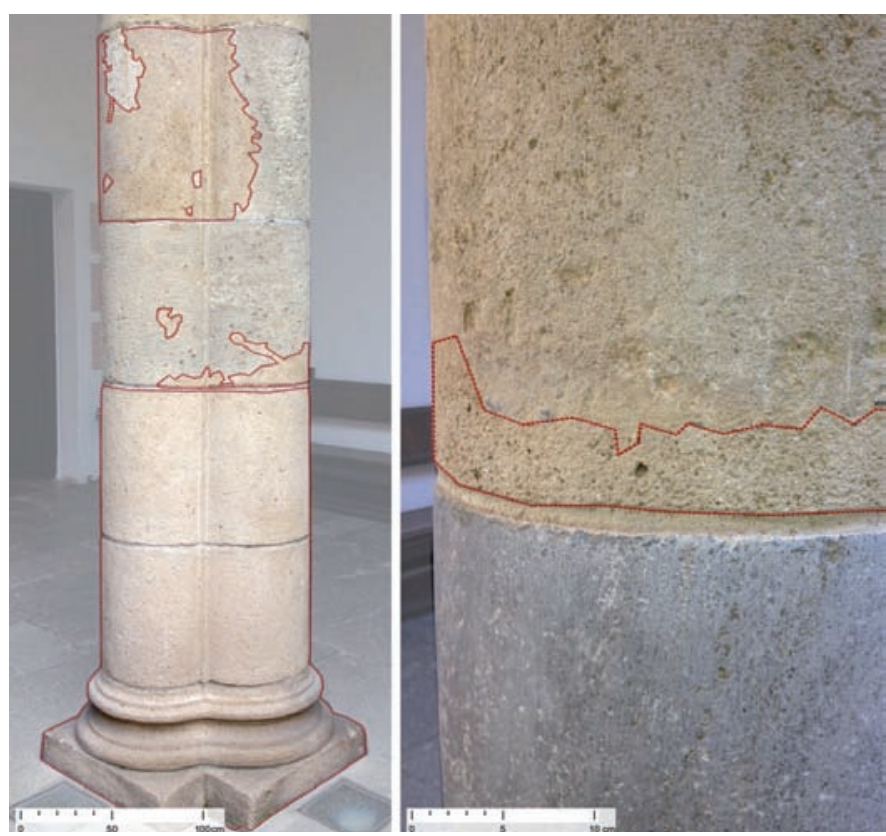

Figura 2. Detalles de piedra calcarenita bioclástica, ubicada en la columnata del Patio de la Capilla del Hospital Real de Granada (s. $\mathrm{XV}$ ) y piedra artificial porosa restauradora en las faltas de soporte. Las líneas quebradas rojas resaltan la piedra artificial porosa añadida como mortero de restauración.

Figure 2. Details of bioclastic calcarenite from the colonnade of the courtyard of the Chapel of the Royal Hospital in Granada (s. XV) and of added restorative porous artificial stone. The broken red lines highlight the porous stone added as restoration mortar. morteros resultantes y relativa rapidez en el fraguado. Para la misma proporción de aglomerante: árido: poliestireno, la adición de cemento blanco como aglomerante se recomienda si se necesitan mejorar las propiedades mecánicas del mortero de restauración resultante (Rezola, 1976; Gomá, 1979).

La adición de poliestireno expandido (EPS) molido a las mezclas no altera las buenas propiedades de plasticidad de las mismas, ya que pueden obtenerse todos los grados de fluidez que se deseen añadiendo cantidades variables de agua, en el caso de aglomerantes inorgánicos, o diluyente, en el caso de resinas orgánicas, lo que garantiza su óptima trabajabilidad en el vertido de coladas en moldes y encofrados o estabilidad de la mezcla al ser aplicada sobre superficies verticales. Sin embargo, pueden utilizarse, según el caso, encofrados o armaduras metálicas o plásticas para mejorar la estabilidad gravitatoria de la masa de mortero para evitar su despegue de las superficies de la piedra, sin que ello suponga algún tipo de desventaja para el fraguado, curado y correcto endurecimiento. Armaduras, mallas o varillas de acero inoxidable (pretensadas o no) pueden ser igualmente necesarias para mejorar la estabilidad mecánica de algunas piezas prefabricadas, particularmente si éstas presentan fuertes diferencias en la razón longitud:anchura:altura, como por ejemplo tableros para enchapados decorativos de paramentos, así como para evitar la aparición de fisuras y grietas no deseables (Martínez, 1967).

La reacción del disolvente orgánico con el poliestireno expandido alojado en la mezcla endurecida se verifica rápidamente siempre que el producto se 
encuentre completamente seco. Esta reacción procede desde la superficie del producto hacia el interior (figura 3), lo que permite una fácil introducción de los reactivos orgánicos a través de la estructura porosa que se está generando, garantizando que puedan acceder a zonas profundas de la reintegración volumétrica y descomponer completamente los granos de poliestireno expandido más inaccesibles, provocando de forma inmediata la estructura porosa pretendida y conectada.

Como ejemplo, la figura 4 muestra la distribución de la porosidad conectada en tres muestras de travertino (de las canteras de Alfacar, Granada) comparada con la porosidad de dos morteros porosos fabricados para simular este tipo de piedra, muy porosa y con mega poros de tamaño centimétrico. Estas mezclas constan de partes variables en volumen. La mezcla para piedra artificial número 3 consta de 1 parte de cal hidráulica, 2 partes de árido fino $(<1 \mathrm{~mm})$ obte- nido por molturación de roca ornamental caliza micrítica de color gris claro, y 3 partes de poliestireno en grano grueso $(3 \mathrm{~mm}-1 \mathrm{~cm}$ ) obtenido por abrasión y triturado mecánico de planchas de poliestireno con densidad de $9 \mathrm{~kg} / \mathrm{m}^{3}$. Todo ello mezclado íntimamente con agua, siguiendo la proporción de 1 I de agua por cada $2 \mathrm{~kg}$ de cal hidráulica. La mezcla para piedra artificial número 4 presenta los mismos componentes que la número 3 , pero con la siguiente proporción: 1 parte de cal hidráulica: 3 árido: 4 poliestireno expandido. La proporción de poliestireno, en ambos casos del $50 \%$ en volumen, es suficientemente elevada para conseguir una porosidad similar o mayor que la del travertino. De hecho, la porosidad en volumen de las muestras de travertino natural es muy variable, con aproximadamente del $30 \%$ de media, mientras que la de la piedra artificial porosa anteriormente mencionada oscila entre el $35-49 \%$. En términos generales el volumen de huecos de la piedra artificial porosa es
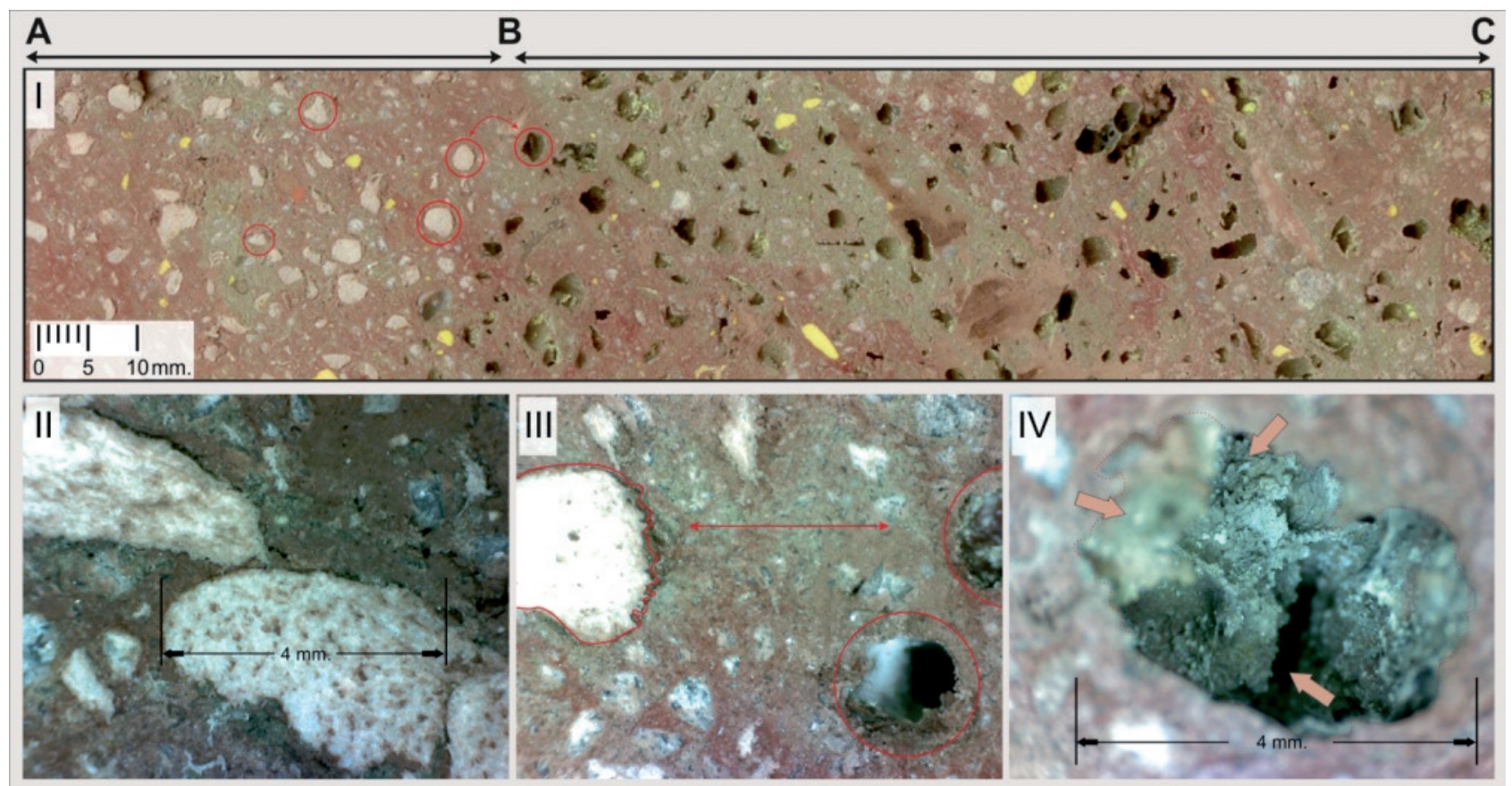

Figura 3. I. Detalle macroscópico de piedra artificial porosa con huecos generados mediante disolución del poliestireno expandido (EPS) triturado. Los círculos en rojo y la flecha curva definen la zona atacada con disolvente. Las líneas superiores con flecha definen en A-B el área donde el generador de poro no ha sido disuelto, mientras que B-C muestra el poro abierto y conectado. La cara externa de la muestra queda a la derecha de la imagen. II. Detalle obtenido con microscopio estereoscópico, donde se pueden observar dos granos de poliestireno expandido sin diluir en una matriz con áridos carbonatados y aglomerante coloreado. III. Detalle de grano de poliestireno expandido sin diluir, marcado por línea quebrada en color rojo, y porosidad conectada tras la dilución del agente generador de poros, marcado por círculo y semicírculo en color rojo. IV. Detalle de hueco con porosidad interconectada. Las flechas definen diferentes profundidades.

Figure 3. I. Macroscopic detail of porous stone with holes generated by dissolution of crushed expanded polystyrene (EPS). Circles in red and the curved arrow define the area reacted after the addition of solvent. The upper lines with arrows in $A-B$ define the area where the pore generator EPS has not been dissolved, whilst B-C shows the open and connected pores. II. Detailed view obtained by binocular microscope, where two grains of reacted EPS are set in a matrix made of aggregates of carbonate and colored binder. III. Detail of unreacted grain of EPS marked by broken red line and connected porosity after the dilution of the pore generator agent, marked by a red circles. IV. Detail of a hollow with interconnected porosity. The arrows define different depths. 


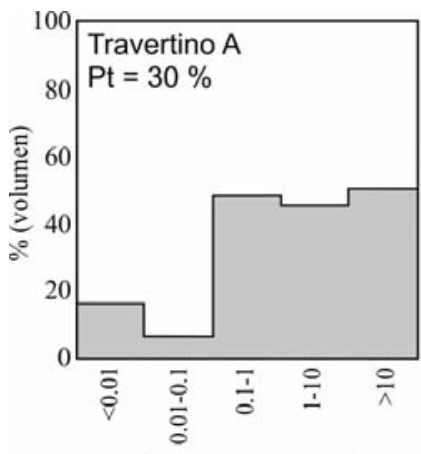

Radio de poro $(\mu \mathrm{m})$

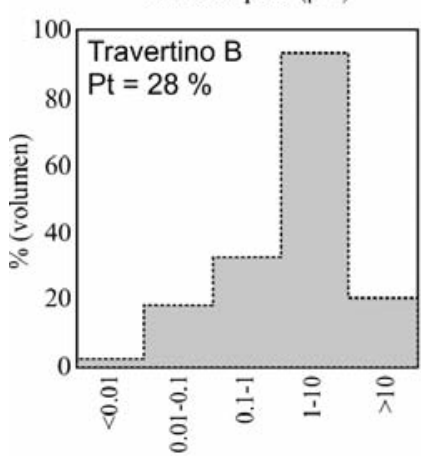

Radio de poro $(\mu \mathrm{m})$

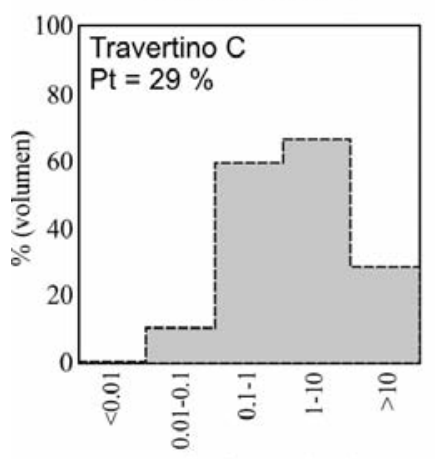

Radio de poro $(\mu \mathrm{m})$

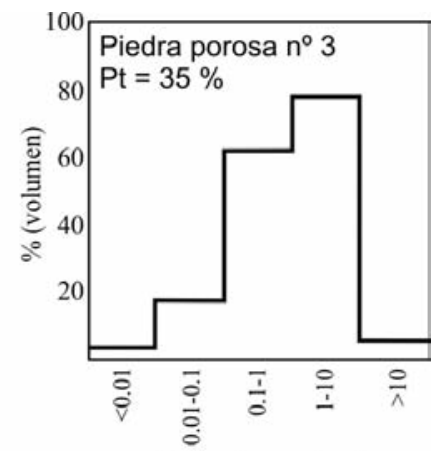

Radio de poro $(\mu \mathrm{m})$

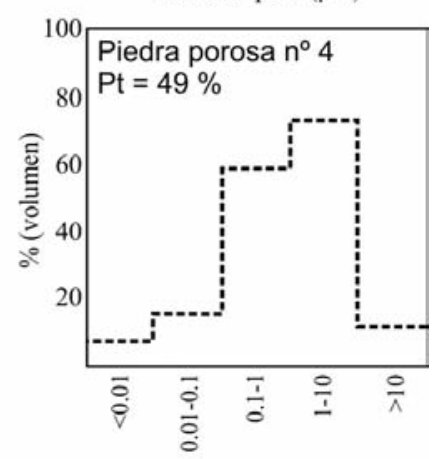

Radio de poro $(\mu \mathrm{m})$

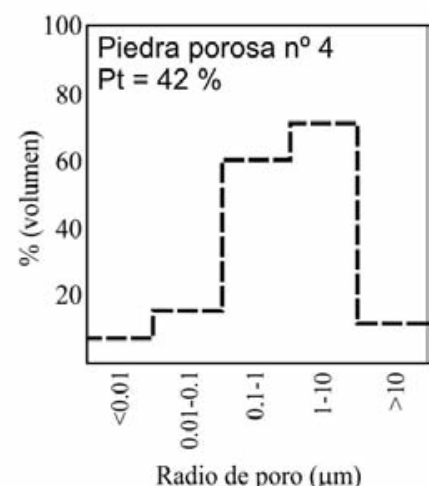

Radio de poro $(\mu \mathrm{m})$

Figura 4. Distribuciones porcentuales de radios de poros en travertino natural procedente de las canteras de Alfacar en Granada y de la piedra artificial porosa aplicable en construcción e intervenciones de restauración (patente con números de publicación ES2187245 A1 y B1 16. 06. 2004). Es apreciable la similitud en la distribución porométrica del producto artificial respecto al natural, resultante de la selección granulométrica del aireante.

Figure 4. Percentage distributions of pore-radii in natural travertine from the quarries of Alfacar in Granada and in porous stone appropriate for construction and restoration work (patent P200001976 (5)). (patent publication ES2187245 A1 and B1 numbers 16. 06. 2004). Note similar porometric distributions, as a result of poregenerator particle size selection.

similar y mayor a la de los travertinos, así como su distribución de tamaños de poro. Este último hecho es capital en la aplicación de la piedra porosa en intervenciones de restauración del patrimonio histórico y artístico expuesto a la intemperie.

Por otra parte, los ensayos de descomposición de poliestireno expandido (EPS) realizados muestran una mayor velocidad de reacción con el tolueno que con otros disolventes. Por ejemplo, las cantidades de reactivo necesarias para descomponer el generador de porosidad son aproximadamente $100 \mathrm{ml}$ de acetona por cada $31 \mathrm{~g}$ de poliestireno (EPS) frente $100 \mathrm{ml}$ de tolueno por cada $51 \mathrm{~g}$ de poliestireno (tabla 1). En ambos casos, la tasa de disolución es más elevada en los primeros instantes de adición del solvente en la piedra artificial porosa, notándose una reducción significativa de la tasa al final del proceso de disolución del generador de porosidad (figura 5). Estas cantidades de disolventes deben entenderse como mínimas al ser aplicadas sobre los productos pétreos artificiales, ya que parte del reactivo se evapora (tabla 1). Por otra parte, en el caso de optar por la aplicación in situ de la piedra artificial porosa, la descomposición del poliestireno al reaccionar con acetona se ve fuertemente inhibido si el producto no está completamente seco. Por todo ello, y para garantizar la descomposición completa del poliestireno alojado en el interior del producto, se recomienda añadir al menos el doble de reactivo que el indicado.

La pérdida del poliestireno expandido en el seno de la masa de roca artificial endurecida no se produce por la aplicación de disolventes: el EPS se repliega hasta niveles importantes, quedando adherido en forma de resina a las paredes de la piedra artificial. La eliminación del poliestireno sí se consigue, en cambio, mediante combustión superficial (que puede llevarse a cabo con soplete o pistola térmica), aunque ello no produce una buena porosidad interconectada necesaria para la utilización de esta roca en intervenciones restauradoras.

Por lo que se refiere a los procesos de alteración de este material una vez puesto en obra, la posibilidad de controlar a voluntad el tamaño de poro final de la piedra artificial porosa simplemente controlando la granulometría del poliestireno es extremadamente beneficiosa, ya que pueden evitarse procesos de deterioro sobre la obra derivados de la infiltración capilar de soluciones acuosas salinas, que ascienden preferencialmente a través de capilares de tamaño fino $(<1 \mathrm{~mm})$. De hecho, la conducción del agua a través de los macroporos abiertos, en contacto con el medio ambiente exterior, permite una rápida evacuación de la misma. Este efecto es beneficioso tanto para utilizaciones del producto con fines estrictamente constructivos como restauradores. Para este último caso, además, una elevada porosidad y la utilización de un aglomerante de baja resistencia garantizan una resistencia mecánica inferior a la de las piedras originales, lo que favorece su degradación preferencial 
Durán Suárez, J.A. y García Casco, A., 2017. Piedra artificial porosa a partir de residuos... Boletín Geológico y Minero, 128 (2): $437-450$

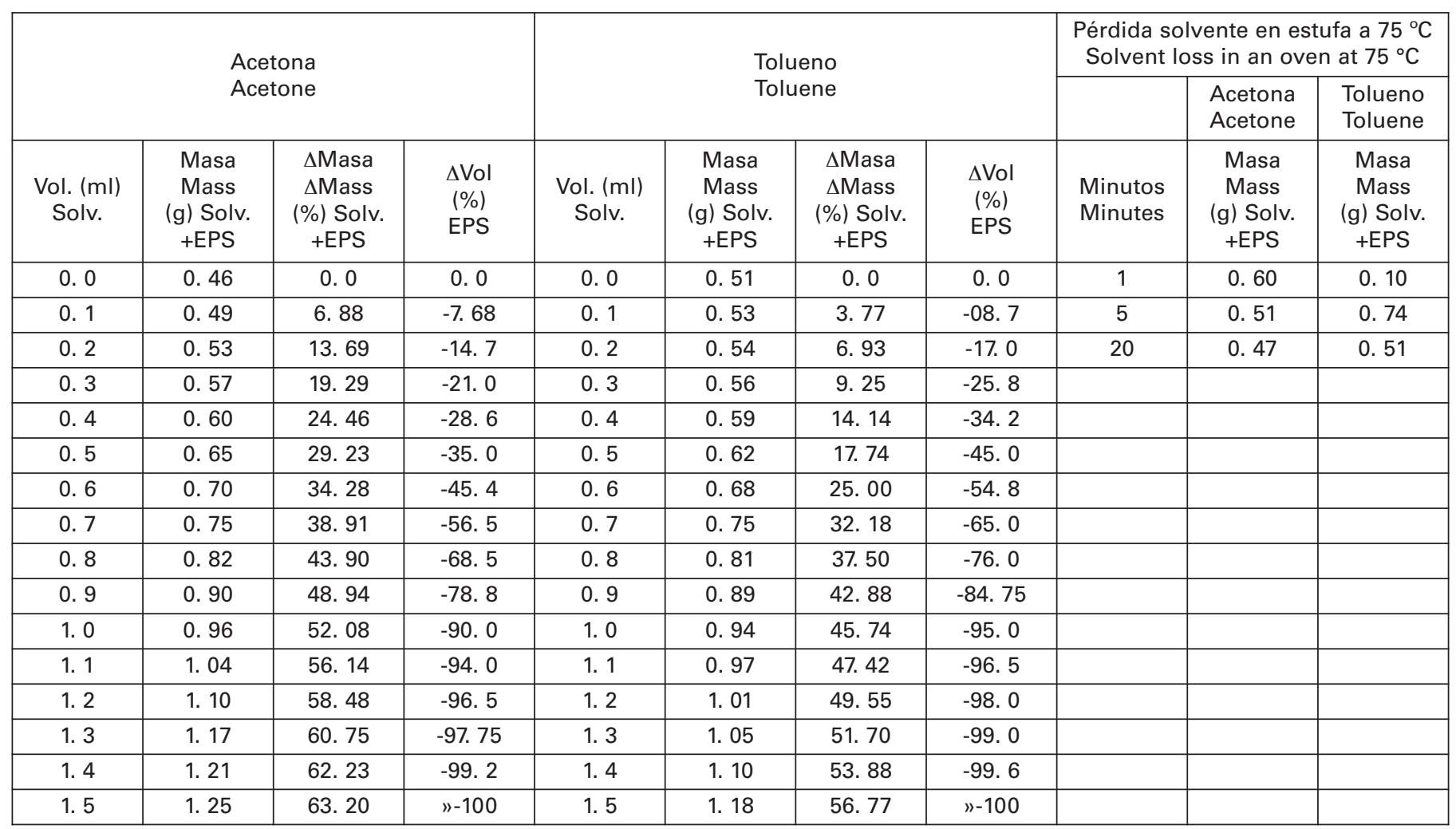

Tabla 1. Valores del ensayo de reactividad del poliestireno expandido con solvente acetona (probeta cúbica de $3 \times 3 \times 3 \mathrm{~cm}$ y peso inicial de $0.46 \mathrm{~g}$ ) y solvente tolueno (probeta cúbica de $3 \times 3 \times 3 \mathrm{~cm}$ y peso inicial de $0.51 \mathrm{~g}$ ). El reactivo se añadió con pipeta calibrada (gotas de 0.1 $\mathrm{ml}$ ) hasta la pérdida total de forma de la probeta de EPS (ver figura 5).

Table 1. Results the dissolution progress of expanded polystyrene in acetone (cubic specimen of $3 \times 3 \times 3 \mathrm{~cm}$ and starting weight of $0.46 \mathrm{~g}$ ) and toluene (cubic specimen of $3 \times 3 \times 3 \mathrm{~cm}$ and starting weight of $0.51 \mathrm{~g}$ ). The solvent was added with a calibrated pipette (drops of $0.1 \mathrm{ml}$ ) up to the loss of the overall shape of the specimen of EPS (see Fig. 5).

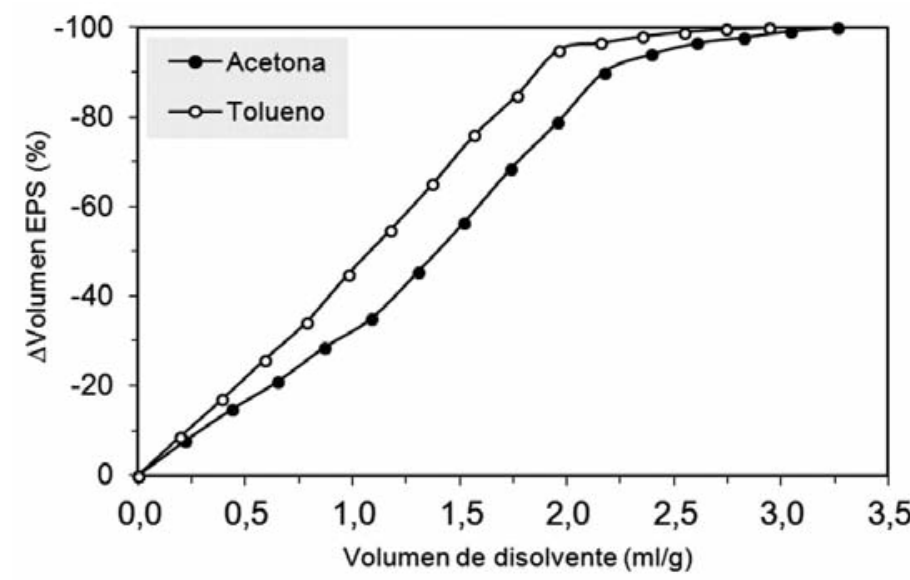

Figura 5. Variación volumétrica de probetas cúbicas de poliestireno expandido (EPS) hasta pérdida total de forma en función de la cantidad de disolvente añadido. Se puede observar una mayor capacidad de disolución del tolueno (verTabla 1).

Figure 5. Volumetric variation of cubic specimens of expanded polystyrene (EPS) until total loss of form as a function of the amount of added solvent. Note the greater dissolution capacity of toluene (see Table 1). respecto de las mismas, hecho este último de capital importancia en la consecución de un mortero de restauración.

La manera de realizar la invención de la piedra artificial porosa es extremadamente sencilla, si bien presenta gran cantidad de variantes en función de las rocas ornamentales a simular y su función constructiva, decorativa o restauradora, dependiendo de las proporciones, naturaleza y tamaño de grano de los componentes de la mezcla. Dejando estas variantes a un lado, fácilmente entendibles, existen dos modos básicos de aplicar la invención: a) como prefabricados, y b) como morteros aplicables en obra (kit adaptable).

\section{Elementos prefabricados.}

En una primera fase, se realizan encofrados o moldes para recibir la mezcla. Una vez disponibles, se proce- 
Durán Suárez, J.A. y García Casco, A., 2017. Piedra artificial porosa a partir de residuos... Boletín Geológico y Minero, 128 (2): $437-450$

de a la fabricación de la mezcla en seco, que constaría de los siguientes pasos:

$1^{\circ}$. Definición de la piedra a simular (en este estudio se asume un travertino veteado).

$2^{\circ}$. Adecuación de la las proporciones de aglomerante, árido, poliestireno y pigmento a la piedra a simular mediante pruebas y ensayos apropiados (resistencia mecánica, porosidad, cromaticidad, etc.). Según nuestros resultados, un travertino puede obtenerse con las proporciones, en volumen, 1:2:3 de aglomerante:árido:poliestireno usando árido de roca ornamental procedente de la industria de la piedra, con tamaño fino $(<1 \mathrm{~mm})$ de naturaleza carbonatada y granos de poliestireno (EPS) gruesos $(1 \mathrm{~mm}, \mathrm{y}$ hasta $1 \mathrm{~cm}$, o mayor). A esta mezcla se le añade 2-5 \% en volumen de aditivos cromóforos a base de tierras naturales, resistentes a los álcalis (Durán, et al. 2000).

$3^{\circ}$. Mezcla en amasadora de las proporciones anteriores y adición de agua con un máximo del 50 $\%$ en volumen de la mezcla total.

$4^{\circ}$. Vertido de la mezcla en estado plástico al molde o encofrado. Este vertido se realiza por tongadas para simular el veteado del travertino simulado. Para ello, se hace necesario preparar más de una mezcla, o mejor aún, modificar ligeramente las tongadas por adicción o reducción de alguno de los componentes aditivos (pigmentos y/o poliestireno expandido), que se irán vertiendo en el orden requerido en cantidades apropiadas para obtener vetas de grosor variable. Lógicamente, esto confiere variaciones texturales y de cromaticidad a las distintas coladas. También es posible variar las proporciones y naturaleza del aglomerante y árido, si bien esto puede introducir fuertes discontinuidades mecánicas en el producto final que pueden ser contraproducentes ante la eventual agresión medioambiental sobre la obra (insolación, percolación de disoluciones acuosas, gelificación, cristalización de sales, etc.).

Si se pretende obtener piezas de gran tamaño, o con grandes diferencias en los valores de longitud, anchura, y altura, es necesaria la instalación de armaduras, mallazos o varillas de acero, preferentemente inoxidable o galvanizados, que pueden pretensarse $o$ no, en los moldes o encofrados antes de proceder al vertido de la mezcla. Por ejemplo, pueden obtenerse tableros utilizando moldes o cajas de dimensiones considerables (varios $\mathrm{m}^{3}$ ), las cuales disponen en su interior de las armaduras justo en los centros de los futuros tableros. Para evitar el corte de bloques, es posible preparar moldes de grandes dimensiones con tabicas múltiples, separadoras de cada uno de los tableros.

Una vez endurecida la mezcla, y siguiendo procedimientos estándar en la tecnología de los hormigones, se procede a hidratar el producto para garantizar un fraguado aceptable. Tras el fraguado inicial, el producto se desmoldea (aproximadamente a las 120 horas) y se cura bajo condiciones de temperatura y humedad adecuadas ( $0{ }^{\circ} \mathrm{C} ;>50 \%$ HR). Opcionalmente, se puede incrementar la resistencia mecánica y acelerar el proceso de endurecimiento mediante el curado en autoclave, es decir, gracias a la introducción de vapor de agua a una presión superior a la ambiental. Conseguidos los valores de resistencia mecánica, el producto manufacturado es retocado, cortado, desbastado y pulido.

A continuación el manufacturado se sumerge, completamente seco, en un baño de disolvente (por ejemplo tolueno) durante unos segundos y se procede a su secado. Finalmente, se puede aplicar un tratamiento superficial con todo tipo de agentes que mejoren o potencien sus propiedades mecánicas (e. g. ésteres del ácido silícico), de superficie (e. g., alquil-aril-polisiloxanos) y estéticas (resinas poliestéricas). Tras este tratamiento, el producto puede ser pulido y abrillantado.

\section{Morteros aplicables en obra (kit adaptable)}

Los morteros adaptables se realizan de la misma manera que los prefabricados, si bien las mezclas se preparan en seco para su posterior amasado en obra. Lo más adecuado es que se suministren desde fábrica con unos valores concretos de aditivo generador de porosidad, resistencia mecánica y cromaticidad. Para este caso, asumiendo la simulación de una arenisca calcárea, la mezcla debe contener las proporciones 1:3:1 de aglomerante:árido:poliestireno expandido (EPS), este último de grano fino y el árido arena gruesa $(1-7 \mathrm{~mm})$ de naturaleza carbonatada. A esta mezcla se añade 2-5\% en volumen de pigmentos a base de tierras naturales (óxidos de hierro, manganeso, etc.). Esta mezcla, lista para su posterior hidratación, puede ser enriquecida con aditivos diversos que confiera mejores características de plasticidad (por ejemplo resinas alquílicas o vinílicas) o viscosidad (sílice coloidal).

El amasado de estas pastas se puede realizar de forma manual o mecánicamente, siguiendo siempre un control riguroso en las herramientas de amasado y en las hormigoneras para que estas no tengan restos de otras pastas utilizadas en la construcción, con lo cual se evita la adición de elementos extraños 
como, por ejemplo, sales solubles, sustancias orgánicas inadecuadas, o agentes pigmentantes inadecuados (Carbajo, 1997). Seguidamente, estas pastas pueden ser aplicadas directamente sobre el paramento deseado, superficie, falta de soporte o laguna, mediante la utilización de espátulas o paletas, o utilizando moldes o encofrados para posteriormente proceder al vertido de las pastas por colada. En estos dos casos, la trabajabilidad de la pasta debe ser diferente: en el primero, el producto tendrá menor plasticidad, consiguiéndose ello aproximadamente a los 15 ó 20 minutos del amasado; en el segundo caso se debe aplicar de manera inmediata para que el mortero tenga la fluidez necesaria para rellenar correctamente el molde o encofrado. Estos dos modos de aplicación son apropiados en el caso que se utilice el mortero como kit de restauración de materiales pétreos (Rossi-Doria, 1986).

Está totalmente desaconsejado el vibrado de estas pastas una vez aplicadas, dado que el agente generador de porosidad (poliestireno expandido EPS) asciende por diferencia de densidad a la superficie de la pasta (Payá, 1985).

El desmoldeado o desencofrado se puede realizar a los pocos días de la colada, siendo conveniente facilitar el curado de las mezclas hidráulicas mediante humectación y riegos periódicos, siempre y cuando no se produzcan heladas. El tiempo de curado y endurecimiento de las mezclas hidráulicas es aproximadamente de un mes; transcurrido este tiempo y una vez se haya secado totalmente se puede proceder a aplicar el reactivo (acetona o tolueno), o bien aplicar calor a la superficie deseada (con soplete o pistola de aire caliente) para descomponer el poliestireno expandido (EPS) contenido en la mezcla, formándose así los poros característicos y novedosos en la piedra artificial porosa (NORMAL 4/80, 1980). Como ventaja adicional cabe destacar la óptima trabajabilidad de la piedra artificial porosa, una vez curada, con todo tipo de herramientas de cantería, tales como: cinceles, gradinas, escofinas, etc, lo que posibilita otorgarle un aspecto textural adecuado con el entorno adyacente.

\section{Conclusiones}

El compuesto artificial consistente en una piedra artificial porosa ha demostrado desde su registro como Patente de invención un excelente comportamiento en obras de construcción y de restauración patrimonial arquitectónica, tanto como elemento prefabricado, como kit adaptable a obra. Sus diferentes reivindicaciones definen el alcance de la protección jurídica y demarcan, de la manera más amplia posible, los límites de la invención con todas sus equivalencias y las probables versiones futuras.

Destaca su inclusión como elementos constructivos con fines decorativos y ornamentales a un coste muy inferior al de la piedra natural, a la par que se reciclan los residuos de la industria de la roca ornamental, dando un uso concreto a este tipo de subproductos tan difícilmente reutilizables. Paralelamente, el gran logro de este manufacturado recae en su uso en actuaciones restauradoras del patrimonio arquitectónico-monumental, fundamentalmente por su sistema de porosidad conectada, gracias a la inclusión de un agente generador de huecos que, tras diversos procedimientos técnicos, se elimina para producir porosidad abierta. Esta porosidad es de capital importancia en las intervenciones de restauración de los soportes pétreos expuestos a la intemperie, pues permite que la humedad alojada en el subsuelo del monumento o del soporte original sea difundida al exterior sin provocar alteraciones mayores.

Las principales reivindicaciones reflejadas en la Patente de invención son las siguientes: piedra artificial porosa caracterizada por estar compuesta de árido, aglomerante, un aditivo generador de porosidad y opcionalmente un aditivo secundario y en el que la estructura porosa es obtenida mediante la descomposición del aditivo generador de porosidad al aplicarse un reactivo. Según reivindicación primera el aditivo generador de porosidad es poliestireno expandido molido (EPS) hasta obtener grano de cualquier tamaño. Según reivindicación primera el reactivo es cualquier producto liquido capaz de penetrar en la mezcla y disolver el aditivo generador de porosidad.

Método para preparar la piedra artificial porosa descrita en las reivindicaciones precedentes que comprende los siguientes pasos: mezclar en seco los componentes; formar una mezcla con los componentes anteriores; adicionar la suficiente cantidad de agua; amasar hasta obtener la consistencia plástica deseada; verter la masa obtenida; esperar el tiempo suficiente para el endurecimiento y secado; generar la estructura porosa mediante la adición del reactivo que disuelve el aditivo generador de porosidad.

Mortero de restauración poroso caracterizado porque se puede preparar de forma idéntica a la piedra de restauración porosa descrita anteriormente y Kit para la preparación de piedra artificial porosa o mortero de restauración poroso descritos anteriormente caracterizado porque consiste en una mezcla en seco de árido, aglomerante, colorante y aditivo generador de porosidad y que posteriormente en la obra solo es necesario añadir agua para elaborar la piedra artificial (Oficina Española de Patentes y Marcas http://invenes. oepm. es). 
Quisiéramos enfatizar, por último, que la utilización de esta piedra artificial porosa es extensa desde hace veinte años en restauraciones patrimoniales de inmuebles con interés histórico y artístico de la ciudad de Granada y su entorno. Su aplicación práctica en el contexto docente de la Licenciatura de Bellas Artes-Restauración y Grado en Conservación y Restauración de Bienes Culturales de la Universidad de Granada, desde el curso académico 1993-94 hasta la fecha, ha facilitado su difusión y empleo por profesionales de la restauración. Destacan las intervenciones restauradoras en los pilares de travertino de periodo Nazarí (s. XV) del patio de la Colegiata del Salvador en Granada, la sillería de calcarenita bioclástica de la Catedral de Granada (s. XVI), los elementos moldurados de calcarenita bioclástica de la fachada del Colegio de S. Pablo de Granada (s. XVII) y la restauración de parte de la columnata de calcarenita bioclástica del Patio de la Capilla del Hospital Real de Granada (s. XVI).

Todas las intervenciones restauradoras anteriormente mencionadas utilizaron la piedra artificial porosa destinada a la restitución y recrecido de las faltas de soporte pétreo original en forma de mortero. En todos los casos, además de la adecuación cromática y textural y resistencia mecánica menor al soporte original, se consiguió que la porosidad del manufacturado añadido fuese conectada y superior a la de la piedra original y con una distribución porcentual de radios de poros muy similar al soporte original subyacente, para facilitar una difusión de agua, en forma de vapor, desde el subsuelo hacia el exterior, evitando fenómenos de ascenso capilar. Particularmente en el caso de la intervención restauradora de la columnata del Patio de la Capilla del Hospital Real de Granada (s. XVI) los resultados de trasmisión de pulsos ultrasónicos (datos inéditos de los autores), tomados longitudinalmente en los fustes de las columnas restauradas con la piedra artificial porosa (Patente P200001976 (5)) y otros morteros no porosos aplicados en restauraciones anteriores, ponen de manifiesto la homogeneidad en la velocidad de trasmisión a lo largo de todo el fuste intervenido con este mortero restitutivo. Esta homogeneidad indica la buena adherencia del mortero poroso de restauración, imitación de piedra calcarenita bioclástica, frente al taponamiento de la humedad subyacente que inducen otros morteros basados en cementos portland, con escasa o nula porosidad, los cuales provocan una alteración visible y no visible del soporte original en forma de arenización y pérdida de resistencia mecánica (por no mencionar su nula integración estética, cromática y textural).

\section{Agradecimientos}

Los resultados de esta investigación se deben a la financiación de los Grupos de Investigación HUM 629 y RNM 302 de la Junta de Andalucía. La titularidad de la Patente pertenece a la Universidad de Granada, siendo los inventores los profesores J. Durán Suárez y A. García Casco de la Universidad de Granada. La tramitación de la patente estuvo a cargo de la Oficina deTransferencia de la Investigación de la Universidad de Granada.

\section{Referencias}

Akbulut, H. and Gürer, C. 2007. Use of aggregates produced from marble quarry waste in asphalt pavements. Building and Environment, 42, 1921-1930.

Arredondo Verdu, F. 1977. Mejora del hormigón mediante su impregnación con polímeros vinílicos: aplicación industrial. Instituto Eduardo Torroja de la Construcción y del Cemento, Madrid, 79 pp.

Asociación Española de Normalización y Certificación. 1996. Áridos para morteros. Definiciones y especificaciones elaboradas por el comité técnico AEN/CTN 146. Madrid, $10 \mathrm{pp}$.

Ashurst, J. and Dimes, G. 1977. Stone in building. The Architectural Press. London, 103 pp.

Carbajo Martínez, M. 1997. Manual de prevención de fallos en los morteros monocapa. Colegio Oficial de Aparejadores y Arquitectos Técnicos de Murcia, Murcia, 67 pp.

Casabó, J. 1958. Fabricación de mosaicos y baldosas de cemento. Nigar, Buenos Aires, 513 pp.

Castro-Gomes, J. P., Almeida, M. D. and Pereira Oliveira, L. A. 2006. Valorization of mining waste on asphalt pavements of low cost. Valorization of Residues Magazine, 3 (9), 9-11.

Castro-Gomes, J. P., Pereira Silva, A., Peralbo Cano, R., Durán Suárez, J. and Alburquerque, A. 2011. Potential for reuse of tungsten mining waste-rock in technical-artistic value added products. Journal of Cleaner Production, 25, 34-41.

Durán Suárez, J. y García Casco, A. 2003. Conservación y restauración de caliza de Sierra Elvira. En: Orfila, M. y Henares, I. (ed), La piedra de Sierra Elvira. Historia y estética. Copartgraf, Granada, 17-29.

Durán Suárez, J., García Beltrán, A., Sáez Pérez, M. and Rodríguez Gordillo, J. 2000. Evaluation of the chromatic effectiveness of color pigments in restoration materials (lime and Portland cement). Color research and application, 25 (4), 286-291.

Esbert, R. y Ordaz, J. 1985. Alterabilidad de la piedra monumental de Laspra (Asturias). Trabajos de Geología, Universidad de Oviedo, 15, pp. 325-331.

Eurostat. 2009. Energy, transport and environment indicators. Waste indicators on generation and land filling measuring sustainable development. 17/12/09 Eurostat Pocketbooks. http://ec. europa. eu/eurostat/docu- 
ments/3930297/5963662/KS-DK-09-001-EN. PDF/ 2ad808 71-2cb9-4784-b781-f15351636352?version=1. 0

Fernández Cánovas, M. 2013. Hormigón. Garceta, Madrid, $672 \mathrm{pp}$.

García Bielsa, J. I. y Gómez Mateo, J. 1995. Tratamiento de lodos de elaboración y aprovechamiento de residuos. En: López Jimeno, C. (ed), Manual de rocas ornamentales. Prospección, explotación, elaboración y colocación. Entorno Gráfico, Madrid, 417-429.

Gomá, F. 1979. El cemento Portland y otros aglomerantes: fundamentos para la interpretación de sus comportamientos en obra. Editores Técnicos Asociados, Barcelona, $232 \mathrm{pp}$.

Hebhoud, H., Aoun, H., Belachia, M. and Ghorbel, E. 2011. Use of marble aggregates in concrete. Construction and Building Materials, 25, 1167-1171.

Jiménez Martínez, R., Álvarez Areces, E., Menduiña, J. and Martín Rubí, J. A. 2009. Materiales utilizados en el patrimonio arquitectónico: la arenisca roja de la catedral de Astorga (León). Boletín Geológico y Minero, 120 (1), 45-52.

Luque Aranda, A. 2011. Andalusian marbles: durability criteria applied in its use as ornamental stone. Tesis Doctoral. Editorial Universidad de Granada. 219 pp.

Marí Bernat, A. 1999. Hormigón armado y pretensado. Universidad Politécnica de Cataluña, Barcelona, 411 pp.

Martínez Ynzenga, J. 1967. Retracción de morteros y hormigones. Instituto EduardoTorroja de la Construcción y del Cemento, Madrid, 73 pp.

Mun, K. J., Choi, N. W., So, S.Y. and Soh,Y. S. 2007. Influence of fine tailings on polyester mortar properties. Construction and Building Materials, 21, 1335-1341.

Murcia Vela, J.; Aguado de Cea, A. and Marí Bernat, A. 1993. Hormigón armado y pretensado. Universidad Politécnica de Cataluña, Barcelona, 584 pp.

NORMAL 4/80. CNR-ICR. 1980. Distribuzione del volume dei pori in funzione del loro diametro. CNR, Roma, $10 \mathrm{pp}$.

Páez Balaca, A. 1986. Hormigón armado. Reverté, Barcelona, 1348 pp.

Payá Peinado, M. 1985. Hormigón vibrado y hormigones especiales. CEAC-Construction, Barcelona, 174 pp.

Peralbo Cano R. 2007. Technical-Sculpture applications of concretes and mortars. Characterization and Standardization. Doctorate Thesis. University of Granada, Spain, 560 pp.

Prado Govea, R.; Louis Cereceda, M; Spairani Berrio, Y, y Huesca Tortosa, J. A. 2009. Estudio y caracterización de morteros para la restauración arquitectónica. XII Congreso de Calidad en la Construcción "CONPAT 2009", Valparaíso-Chile, 145-154.

Rezola Izaguirre, J. 1976. Características y correcta aplicación de los diversos tipos de cemento: Portland, siderúrgicos, puzolánicos, aluminosos, compuestos y naturales. Editores Técnicos Asociados, Barcelona, 152 pp.
Rodríguez Navarro, C. 1994. Causas y mecanismos de alteración de los materiales calcáreos de las catedrales de Granada y Jaén. Tesis Doctoral. Editorial Universidad de Granada. 412 pp.

Rossi-Doria, P. R. 1986. Mortars for restoration: basic requirements and quality control. Materiaux et constructions. 19 (114), 445-448.

Russell, S. A. 1927. Stone Preservation Committee Report (Appendix I). HMSO, London.

Santiago Godos, V. 1995. La restauración de la piedra natural. En: López Jimeno, C. (ed), Manual de rocas ornamentales. Prospección, explotación, elaboración y colocación. Entorno Gráfico, Madrid, 549-582.

Torgal, F. P., Castro-Gomes, J. P. and Jalali, S. 2009. Utilization of mining wastes. En: John, Van Deventer, J. (ed), Geopolymers: Structure, Processing, Properties and Applications. Woodhead Publishing, Amsterdam, 235-562.

Torgal, F. P., Castro-Gomes, J. P. and Jalali, S. 2008. Properties of tungsten mine waste geopolymeric binder. Construction and Building Materials 22, 1201-1211.

UNE-EN 196-1:2005 Métodos de ensayo de cementos. Parte 1: determinación de resistencias mecánicas.

UNE 127748-2:2006 Baldosas de terrazo. Parte 2: Baldosas de terrazo para uso exterior.

UNE 127748-1:2006 Baldosas de terrazo. Parte 1: Baldosas de terrazo para uso interior.

Universidad de Granada Piedra artificial porosa: prefabricados y morteros adaptables (kit) para aplicación en obras de construcción y restauración. Inventores: Durán Suárez, J. A. y García Casco, A. Int. Cl. 7: C04B 38/0. España, patente de invención, ES 2187245 A1 y B1. 16. 06. 2004.

Veniale, F. and Zezza, U. 1988. Nuove indagini sull arenaria della Basilicata di S. Michelle in Pavia. Atti Ticinensi de Scienze della Terra, 31,253-268.

Vénuat, M. 1972. Aditivos y tratamientos de morteros y hormigones. Editores Técnicos Asociados, Barcelona, 416 pp.

Weigler, H and Sieghart, Karl. 1974. Hormigones ligeros armados. Gustavo Gili, Barcelona, 261 pp.

Yellishetty, M., Karpe, V., Reddy, E. H. and Subhash, K. N. 2008. Reuse of iron ore mineral wastes in civil engineering constructions: a case study. Resources, Conservation and Recycling, 52, 1283-1289.

Zehnder, K. 1982. Verwitterung von Molassesandsteinen an Bauwerken und in Naturaufschlüssen. Beiträge zur Geologie der Schweiz Geotechnische, 61, 130 pp.

Zhang, L., Ahmari, S. and Zhang, J. 2011. Synthesis and characterization of fly ash modified mine tailings-based geopolymers. Construction and Building Materials, 25, 3773-3781.

Recibido: diciembre 2015

Revisado: febrero 2015

Aceptado: abril 2016

Publicado: junio 2017 